\title{
Uncertainty Assessment of Vessels Width Measurement from Intensity Profile Model Fitting in Fundus Images
}

\author{
Fantin Girard ${ }^{1}$, Conrad Kavalec ${ }^{2}$, Houssem Ben Tahar ${ }^{3}$, and Farida Cheriet ${ }^{1}$ \\ 1 École Polytechnique de Montréal, Montreal, QC H3C 3A7, Canada, \\ fantin.girard@polymtl.ca \\ 2 St.Mary's Ophthalmology Clinic, Montreal, QC H3T 1M5, Canada, \\ conrad .kavalec@mcgill. ca \\ 3 DIAGNOS Inc., Brossard, QC J4Z 1A7, Canada
}

\begin{abstract}
Arteriolar-to-venular diameter ratio (AVR) is an important clinical measurement that allows to characterize retinal vascular abnormalities. A reliable AVR measurement requires accurate and reproducible width measurement. However, in order to measure the vessel width automatically, an approximation of the intensity profile is required by fitting a model. The aim of the proposed study is to assess the uncertainties introduced in the vessel width measurements when choosing a specific distribution as an intensity profile model. Different models are described and an automatic vessel width measurement procedure is presented. The uncertainty introduced by each model is evaluated by computing the standard deviation of the difference between the automatic and the manual measurements. The results show that the intensity profile model should be chosen according to the relative width of the targeted vessels.
\end{abstract}

Keywords: vessel width measurement, retinal photography, vessel model.

\section{Introduction}

Arteriolar-to-venular diameter ratio (AVR) is an important clinical criteria that can be used to predict many diseases like stroke, myocardial infarct, diabetic retinopathy [1][2]. The AVR is defined as the ratio between arteries and veins near the optical disk area and a clinical protocol has been described in [3]. Changes in the AVR are really difficult to detect in fundus images without an automatic process to assist the specialists. An automated process consists in reproducing the manual measurements but it requires the optical disk to be detected, the retinal blood vessels to be segmented, their width to be measured and finally arteries and veins to be classified [4]. In addition, the largest veins and arteries near the optical disk are used to calculate the AVR. The precision of the width measurement is critical for a reliable AVR. One way of calculating a vessel width is to fit a model to its intensity profile. Thus the estimation of the width is more robust to noise. Several models have been presented for fitting 
retinal vessel intensity profile. Several 1D profile models have been proposed in the litterature: step gate [5], Gaussian [6], mixture of Gaussians [7], Laplacian [8] and Hermite [9][10]. The first two models are simple and widespread, but rather approximate. The three others are more realistic by taking into account light reflections, yet they are more involving since they embed more parameters to estimate. These 1D models can be extended to 2D models by assuming that locally vessels width are not changing drastically along the vessels. The purpose of this paper is to study the impact of the intensity profile model on the width measurement and therefore on the AV ratio estimation. In this work, we present different functions that can be used to model the vessel intensity profiles. Then a methodology to measure width automatically is described. Finally, we show the results of the width measurement algorithm obtained with the different models on the vessels extracted from three public databases.

\section{Retinal Vasculature Intensity Profile Models}

Before introducing the width measurement procedure, we present different functions that can be used to model the vessels. A sample vessel profile is given in Fig.1

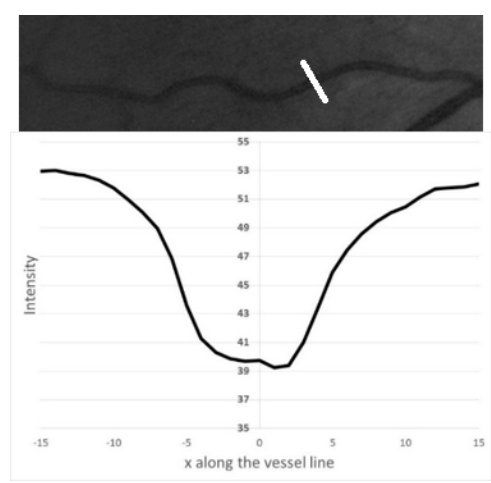

Fig. 1. On top a vessel segment from INSPIRE-AVR [4], on the bottom the vessel profile along the normal

\subsection{Gaussian Model}

A Gaussian intensity model is defined by:

$$
I(x)=t-h e^{\frac{-1}{\sigma^{2}}(X-\mu)^{2}}
$$

Where $x$ is the coordinate along the normal of the vessel profile; $t$ the maximum intensity profile, $h$ the maximum height of the first Gaussian, $\sigma^{2}$ the 
variance of the first Gaussian, $\mu$ the offset of the Gaussian center from the centerline estimate.

If we define the width to be proportional to the difference between the two inflexion points, it can be simply expressed as:

$$
w=\sqrt{2} \sigma
$$

\subsection{Hermite Model}

The Hermite distribution is more flexible than the Gaussian distribution as it can model the light reflection with only one more parameter $\beta$. The Hermite distribution can be written as follows:

$$
I(x)=t-h\left(1+\beta\left((X-\mu)^{2}-1\right)\right) e^{\frac{-1}{\sigma^{2}}(X-\mu)^{2}}
$$

Considering the width as the difference between the two inflection points, we can calculate it as follows:

$$
w=\sqrt{\frac{1}{2 \beta}\left[10 \beta \sigma^{2}-4+4 \beta+2 \sqrt{\beta^{2}\left(17 \sigma^{4}+12 \sigma^{2}+4\right)+\beta\left(-8-3 \sigma^{2}\right)+4}\right]}
$$

The width $w$ depends on two parameters $\beta$ and $\sigma$. When $\beta$ increases the width decreases and on the contrary when $\sigma$ increases the width increases.

\subsection{The Generalized Normal Distribution (GND) Model}

We introduce a new model not used in the literature for retinal vessel profile approximation. It can model wider peaks around $\mu$ which are observed in fundus images.

$$
I(x)=t-h e^{\frac{-1}{\sigma^{n}}(X-\mu)^{n}}
$$

As the Hermite model, it involves five parameters. The width can be simply expressed as:

$$
w=2\left(\frac{n-1}{n}\right)^{\frac{1}{n}} \sigma
$$

\section{Automatic Width Measurement}

\subsection{Preprocessing}

A width measurement algorithm has been developed to compare the performance of the different models (see on Figure 2).

As in [11], we first perform a segmentation algorithm, then the Zhang-Suen thinning algorithm [12] is used. We use the hit-and-miss morphological operation to prune spurs due to the thinning algorithm. Bifurcations and crossings are found in order to divide the vessel network into segments once again using a hit-and-miss morphological operation. The normal and orientation at each point of the segment are found using a PCA in the neighborhood of the considered point. 


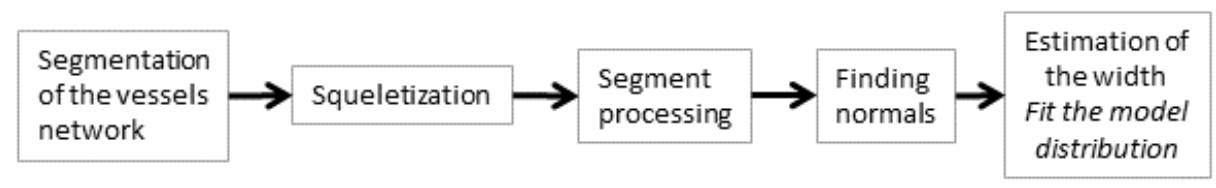

Fig. 2. Automatic width measurement algorithm

\subsection{Vessel Width Measurement}

To reduce the searching space, the two maximas of the gradient along the normal and away from the centerline are found. The difference between these two maximas gives a first estimate of the width.

The second estimate uses the a priori information that the width of the vessels do not change drastically along the segment and that the profile follows the chosen distribution model. To take into account these a priori, the mean $I_{o b s}(X)$ of $N$ intensity observations $I_{Y}(X)$ are calculated for each point on the vessel segment (see on Figure 3). $X$ is the coordinate along the normal of the segment.

$$
I_{o b s}(X)=\frac{1}{N} \sum_{Y={ }^{N} / 2}^{N / 2} I_{Y}(X), \quad N=10
$$

The model parameters are then found so that the residual error $e$ is minimized, where $I$ is one of the model function seen in Section 2.

$$
e(\sigma, h, t, \mu)=\sum_{X=-50}^{50}\left(I(X, \sigma, h, t, \mu)-I_{o b s}(X)\right)
$$

Then the width is calculated using the equations seen in Section 2 depending on the chosen model.

\section{Results}

Three public databases have been used for the evaluation experiments: INSPIREAVR [4], REVIEW [13], and DRIVE [14] databases. These databases have different resolutions. For the DRIVE database, we rely on the given manual segmentation. For the two other databases, we used an automatic line detector-based segmentation.

\subsection{Comparison of the Vessel Profile Models}

From these databases, 1000 vessels segments have been extracted and the different models have been fitted to their intensity profiles. The intensity residual 


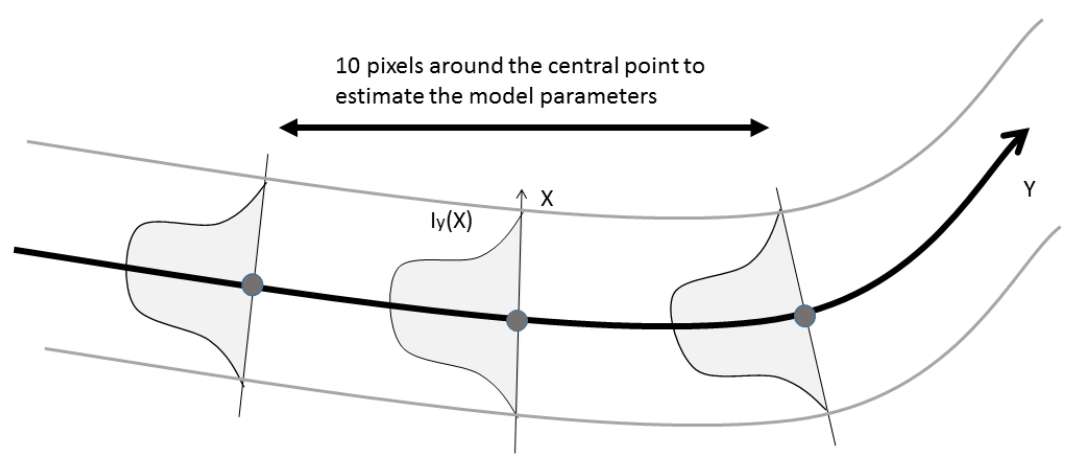

Fig. 3. Intensity profile model fitting procedure

error is an indicator of how the model is well fitted to the observation. Figure 4 shows that when the Gaussian model create a high residual error. On the contrary, Hermite model has the smallest residual error on large vessels mainly because it performs better on vessels with light reflection.

In addition, Figure 5 shows two profiles: $a$ ) one profile with steeper shape where the GND is better than other models; $b$ ) one profile with light reflection where the Hermite distribution is better.

\subsection{Precision of the Vessel Width Measurement}

REVIEW database contains fourteen fundus images divided in three datasets (CLRIS, HRIS and VDIS) from which vessels were manually measured by three experts. The ground truth measurement is defined as the average of the three width values provided by the three experts. The precision of the width performance algorithm is evaluated by calculating the standard deviation $\sigma_{\chi}$ of the width differences $\chi_{i}$ between the automatic and manual measurement, respectively $\omega_{i}$ and $\psi_{i}[13]$.

$$
\chi_{i}=\omega_{i}-\psi_{i}
$$

The generalized normal distribution has he smallest variance when looking at the global standard deviation for each datasets of REVIEW (see on Table 1). If the standard deviation is calculated as a function of the width (see Figure 6 ), then it appears that there are different models that minimize the variance depending on the width.

The distribution model should be chosen in accordance to the width as shown in Table 2.

Using a Gaussian assumption can lead to inaccurate width estimation on large vessels and to a false AR ratio as it is calculated on large vessels present in the area around the optic disk. 


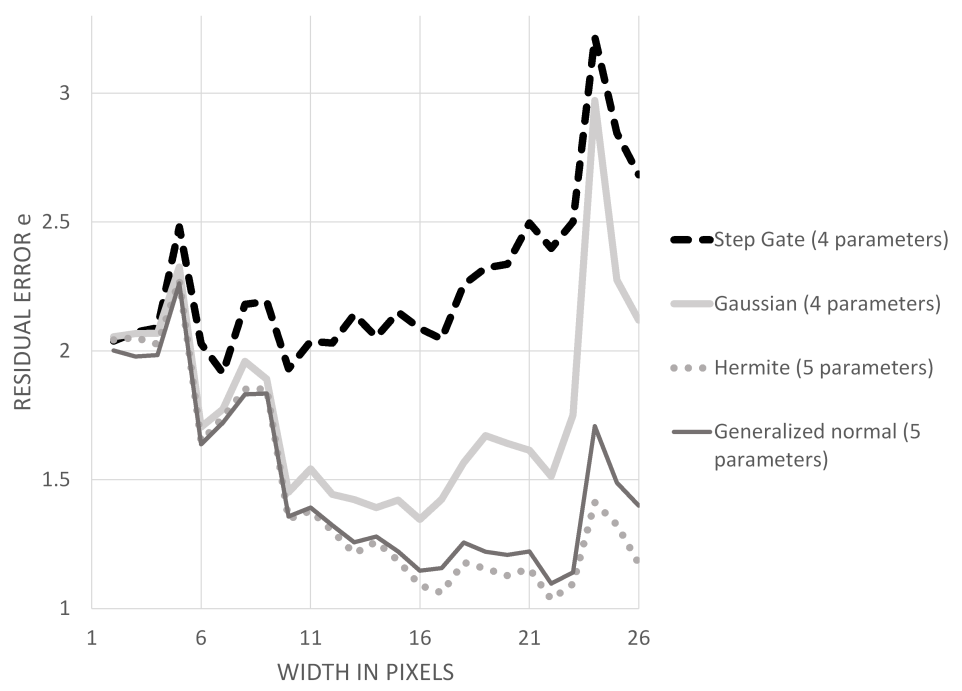

Fig. 4. Intensity residual error for different models with respect to vessel widths

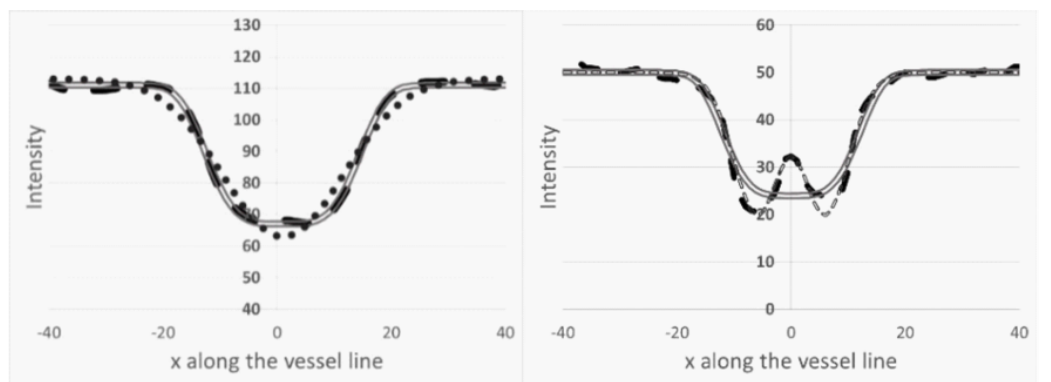

Fig. 5. Left: Generalized normal model (GND) is better than other models, dash line: observation, dot line: Gaussian, double line: GND. Right: Hermite model is better than GND in presence of light reflection, dash line: observation, grey dash line: Hermite, double line: GND

Table 1. Standard deviation $\sigma_{\chi}$ of the width differences $\chi_{i}$

\begin{tabular}{|c|c|c|c|}
\hline & CLRIS & HRIS & VDIS \\
\hline Expert 1 & 0.59 & 0.75 & 0.44 \\
Expert 2 & 0.65 & 0.61 & 0.44 \\
Expert 3 & 0.71 & 0.77 & 0.53 \\
Step-Gate & 1.83 & 2.04 & 3.85 \\
Gaussian & 1.88 & 2.58 & 3.38 \\
GND & 1.69 & 2.02 & 3.18 \\
Hermite & 1.83 & 2.19 & 3.49 \\
\hline
\end{tabular}




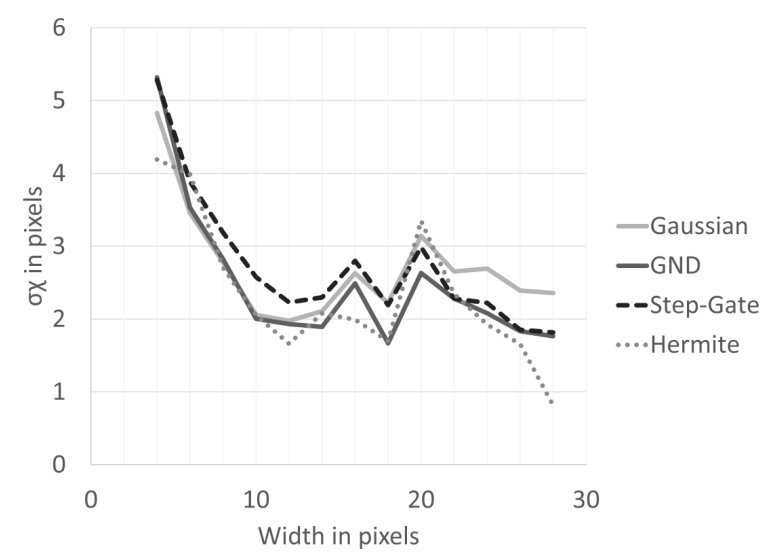

Fig. 6. $\sigma_{\chi}$ with respect to the width for different distributions

Table 2. Optimal distribution profile according to the vessel width

\begin{tabular}{|c|c|}
\hline WIDTH (in pixels) & Best distribution \\
\hline From 4 to 10 & Gaussian \\
\hline From 10 to 18 & Hermite \\
\hline From 18 to 23 & GND \\
\hline Superior to 23 & Hermite \\
\hline
\end{tabular}

\section{Discussion}

The generalized model does not handle the light reflection. This model could be modified to add a smaller Gaussian but it would increase the number of parameters. In our opinion, the best way to overcome this problem is to combine two simple models like Hermite and Generalized Normal models and decide which one is the best based on the intensity residual error.

There are still challenges to estimate a model for small vessels (inferior to 5 pixels) because of the resolution.

Fitting a model is still difficult in presence of low contrast when the cup of the vessel profile and the background blend together.

\section{Conclusion}

This study shows that the model have to be chosen depending on the expected vessel width. Using the residual error as an indicator of performance will allow us to combine different models and to improve vessel width measurement precision. Further work should be done to combine the different models and to assess the performance on AR ratio measurements. 
Acknowledgments. The authors wish to acknowledge the financial support from MEDITIS biomedical program and from the Natural Sciences and Engineering Research Council of Canada (NSERC).

\section{References}

1. Sun, C., Wang, J.J., Mackey, D.A., Wong, T.Y.: Retinal vascular caliber: Systemic, environmental, and genetic associations. Survey of Ophthalmology 54(1) (2009) $74-95$

2. Abramoff, M.D., Garvin, M.K., Sonka, M.: Retinal imaging and image analysis. Biomedical Engineering, IEEE Reviews in 3 (2010) 169-208

3. Hubbard, L.D., Brothers, R.J., King, W.N., Clegg, L.X., Klein, R., Cooper, L.S., Sharrett, A.R., Davis, M.D., Cai, J.: Methods for evaluation of retinal microvascular abnormalities associated with hypertension/sclerosis in the atherosclerosis risk in communities study. Ophthalmology 106(12) (1999) 2269-80

4. Niemeijer, M., Xu, X., Dumitrescu, A.V., Gupta, P., van Ginneken, B., Folk, J.C., Abramoff, M.D.: Automated measurement of the arteriolar-to-venular width ratio in digital color fundus photographs. IEEE Trans Med Imaging 30(11) (2011) 1941-50

5. Chutatape, O., Liu, Z., Krishnan, S.M.: Retinal blood vessel detection and tracking by matched gaussian and kalman filters. In: Engineering in Medicine and Biology Society, 1998. Proceedings of the 20th Annual International Conference of the IEEE. Volume 6. 3144-3149 vol.6

6. Liang, Z., Rzeszotarski, M.S., Singerman, L.J., Chokreff, J.M.: The detection and quantification of retinopathy using digital angiograms. Medical Imaging, IEEE Transactions on 13(4) (1994) 619-626

7. Lowell, J., Hunter, A., Steel, D., Basu, A., Ryder, R., Kennedy, R.L.: Measurement of retinal vessel widths from fundus images based on 2-d modeling. IEEE Trans Med Imaging 23(10) (2004) 1196-204

8. Vermeer, K.A., Vos, F.M., Lemij, H.G., Vossepoel, A.M.: A model based method for retinal blood vessel detection. Comput Biol Med 34(3) (2004) 209-19

9. Li, W., Bhalerao, A., Wilson, R.: Analysis of retinal vasculature using a multiresolution hermite model. Medical Imaging, IEEE Transactions on 26(2) (2007) $137-152$

10. Lupascu, C.A., Tegolo, D., Trucco, E.: Accurate estimation of retinal vessel width using bagged decision trees and an extended multiresolution hermite model. Med Image Anal 17(8) (2013) 1164-80

11. Xiayu, X., Niemeijer, M., Qi, S., Sonka, M., Garvin, M.K., Reinhardt, J.M., Abramoff, M.D.: Vessel boundary delineation on fundus images using graph-based approach. Medical Imaging, IEEE Transactions on 30(6) (2011) 1184-1191

12. Zhang, T.Y., Suen, C.Y.: A fast parallel algorithm for thinning digital patterns. Commun. ACM 27(3) (1984) 236-239

13. Al-Diri, B., Hunter, A., Steel, D., Habib, M., Hudaib, T., Berry, S.: Review a reference data set for retinal vessel profiles. In: Engineering in Medicine and Biology Society, 2008. EMBS 2008. 30th Annual International Conference of the IEEE. 2262-2265

14. Staal, J., Abramoff, M.D., Niemeijer, M., Viergever, M.A., van Ginneken, B.: Ridge-based vessel segmentation in color images of the retina. Medical Imaging, IEEE Transactions on 23(4) (2004) 501-509 